\title{
Convolution surfaces of quadratic triangular Bézier surfaces
}

\author{
Martin Peternell Boris Odehnal
}

March 8, 2007

\begin{abstract}
In the present paper we prove that the polynomial quadratic triangular Bézier surfaces are LN-surfaces. We demonstrate how to reparameterize the surfaces such that the normals obtain linear coordinate functions. The close relation to quadratic Cremona transformations is elucidated. These reparameterizations can be effectively used for the computation of convolution surfaces.
\end{abstract}

Keywords: Quadratic Bézier triangle, LN-surface, reparameterization, Cremona transformation, convolution surface.

\section{Introduction}

Quadratic triangular Bézier surfaces are a well explored area, and many textbooks on CAGD, e.g. [6, 8] are covering this topic. Thus one may wonder what else can be said about them? Surprisingly, in connection with the computation of convolution surfaces it has been proved recently in [12] that the convolution surfaces of quadratic triangular Bézier surfaces and any arbitrary rational surface are always rational. This result is quite surprising, since the rationality condition for the convolution surfaces is rather strong. One direct consequence is that the offset surfaces of quadratic triangular Bézier surfaces are rational surfaces. Unfortunately [12] does not disclose the geometric properties being responsible for the rationality of the convolution surfaces.

Earlier it has been proved in [23] that the convolution surface of an LN-surface and an arbitrary rational surface is always rational. LN-surfaces have been introduced in [9] and denote a special class of surfaces whose normal vectors admit a linear parameterization depending on the surface parameters. 
The main contribution of this article is to prove with geometric reasons that quadratic triangular Bézier surfaces are $L N$-surfaces and to study the geometric background of this property. This brings the result of [12] in connection to what has been proved in [23]. This property is not immediately seen from the standard parameterization, but geometric considerations concerning the structure of the family of their tangent planes lead to reparameterizations proving this fact. These special parameterizations simplify the computation of convolution surfaces.

The notion of convolution appears in two different ways: In computer graphics a convolution surface is defined as level set of an implicit function $f(\mathbf{x})=g(\mathbf{x}) \star h(\mathbf{x})=$ $\int_{\mathbb{R}^{3}} g(\mathbf{t}) h(\mathbf{x}-\mathbf{t}) d \mathbf{t}$, called the convolution of the geometry function $g$ and the kernel function $h$, see $[3,17,26]$.

In geometric modeling the convolution surface of two surfaces $F$ and $G$ in Euclidean threespace $\mathbb{R}^{3}$ is defined by

$$
F \star G=\left\{\mathbf{f}+\mathbf{g} \mid \mathbf{f} \in F, \mathbf{g} \in G, \mathbf{n}_{F} \| \mathbf{n}_{G}\right\},
$$

where $\mathbf{f}(u, v)$ and $\mathbf{g}(s, t)$ are the respective parameterizations and $\mathbf{n}_{F}$ and $\mathbf{n}_{G}$ are the respective normal vectors of $\mathbf{f}$ and $\mathbf{g}$, see e.g. $[13,14,16]$.

Geometrically speaking one forms the sum $\mathbf{f}+\mathbf{g}$ of vectors for those points whose normal vectors are parallel. Considering $G$ as family of translations, the convolution surface $F \star G$ is the envelope of $F$ under the translations defined by vectors $\mathbf{g}$. In general we cannot expect that $F$ and $G$ are parameterized in a way that their normal vectors $\mathbf{n}_{F}=\mathbf{f}_{u} \times \mathbf{f}_{v}$ and $\mathbf{n}_{G}=\mathbf{g}_{s} \times \mathbf{g}_{, t}$ are parallel for $(u, v)=(s, t)$. Typically it is necessary to reparameterize one of the input surfaces, say $G$.

Assume that $\mathbf{f}(u, v)$ and $\mathbf{g}(s, t)$ are rational parameterizations. The question arises in which cases the convolution surface $F \star G$ again admits a rational parameterization. It turned out in [18] and [23] that $F \star G$ is rationally parameterized if $F$ is a paraboloid or more general an LN-surface and $G$ is any rational surface. Earlier it was shown in [16] that two rational skew ruled surfaces $F$ and $G$ always yield a rational convolution surface $F \star G$. These results already indicate that the rationality of the convolution surface is in close relation to the structure of the families of tangent planes of $F$ and $G$.

This topic is related to the question which rational surfaces possess rational offset surfaces. Surfaces with rational offsets are called $P N$-surfaces and their unit normal vectors are a rational parameterization of the Euclidean unit sphere $S^{2}$. An explicit construction of these surfaces has been given in [20] and several surprising examples are given in [19]. Rational offsets of LN-surfaces are discussed in [10]. An approach using classical geometries for NURBS curves and surfaces is presented in [21].

The paper is organized as follows: Section 2 explains some geometric properties of LNsurfaces, quadratic triangular Bézier surfaces, the Veronese surface $V_{2}^{2}$ and its projections. In Section 3 we discuss the dual representation of quadratic triangular Bézier surfaces 
and the computation of base points is explained too. After that planar quadratic Cremona transformations follow in Section 4, which are the key to the reparameterization. In Section 5 we give a proof of the LN-property of quadratic triangular Bézier surfaces. A synthetic proof of this property can already be found in Section 2.4. Section 6 contains examples of the reparameterization and of the convolution surface of two quadratic triangular Bézier surfaces. Finally we conclude in Section 8.

\section{Geometric background}

Points in $\mathbb{R}^{3}$ are represented by their coordinate vectors $\mathbf{x}=(x, y, z)$. The projective closure of $\mathbb{R}^{3}$ is denoted by $\mathbb{P}^{3}$ and points in $\mathbb{P}^{3}$ are identified with their homogeneous coordinate vectors

$$
\mathbf{x} \mathbb{R}=\left(x_{0}, x_{1}, x_{2}, x_{3}\right) \mathbb{R}=\left(x_{0}: x_{1}: x_{2}: x_{3}\right), \text { with } \mathbf{x} \neq \mathbf{o} .
$$

Choosing the plane at infinity $\omega$ as $x_{0}=0$, the interchange between homogeneous and Cartesian coordinates for points in $\mathbb{R}^{3}$ is realized by

$$
x=\frac{x_{1}}{x_{0}}, \quad y=\frac{x_{2}}{x_{0}}, \quad z=\frac{x_{3}}{x_{0}} .
$$

Similarly we use $(u, v)$ as affine coordinates in the parameter plane $\mathbb{R}^{2}$ and $\left(u_{0}: u_{1}: u_{2}\right)$ as homogeneous coordinates in the projective closure $\mathbb{P}^{2}$ of $\mathbb{R}^{2}$. The analogous relation between affine and homogeneous parameters reads

$$
u=\frac{u_{1}}{u_{0}}, \quad v=\frac{u_{2}}{u_{0}} .
$$

Moreover, we have to consider the dual projective space $\mathbb{P}^{3 \star}$, whose points are identified with the family of planes in $\mathbb{P}^{3}$. Let $e_{0}+e_{1} x+e_{2} y+e_{3} z=0$ be the equation of a plane $E$. The homogeneous coordinate vector $\mathbb{R} \mathbf{e}=\mathbb{R}\left(e_{0}, e_{1}, e_{2}, e_{3}\right)$ is identified with $E$. If especially $E: h+u x+v y+z=0$ is given as graph of a linear function, we may use the affine coordinates $(h, u, v)$ of $E$ too.

\section{$2.1 \quad$ LN-surfaces}

A rational surface $S$ is called an $L N$-surface if there exists a rational parameterization $\mathbf{s}(u, v)$ such that a normal vector field $\mathbf{n}(u, v)$ of $S$ can be linearly parameterized as

$$
\mathbf{n}(u, v)=\mathbf{p} u+\mathbf{q} v+\mathbf{r},
$$

where $\mathbf{p}, \mathbf{q}$, and $\mathbf{r}$ are vectors in $\mathbb{R}^{3}$. A particular parameterization $\mathbf{s}(u, v)$ of $S$ may not show the LN-property (4) at once. A rational reparameterization may be required. 
Depending on the rank of $M:=(\mathbf{p}, \mathbf{q}, \mathbf{r})$ we have to distinguish the following cases: If rk $M=1, S$ is a part of a plane and if rk $M=2$ the unit normals $\mathbf{n}_{0}=\mathbf{n} /\|\mathbf{n}\|$ of $S$ are contained in a great circle on the Euclidean unit sphere $S^{2}$. This implies that $S$ is contained in a cylinder.

In the following we assume rk $M=3$. This implies that the unit normal vectors of $S$ parameterize a two-dimensional subset of $S^{2}$. An appropriate choice of the coordinate system yields

$$
\mathbf{p}=(1,0,0)^{T}, \quad \mathbf{q}=(0,1,0)^{T}, \quad \mathbf{r}=(0,0,1)^{T},
$$

and the normal vector simplifies to $\mathbf{n}(u, v)=(u, v, 1)^{T}$. Thus the tangent planes $T(u, v)$ of an LN-surface allow the quite simple representation

$$
T(u, v): h(u, v)+u x+v y+z=0,
$$

where $h(u, v)$ is a rational function (support function of $T$ ). With respect to the chosen coordinate system, the tangent planes $T(u, v)$ are graphs of linear functions over the $[x, y]$ plane. The representation (5) allows to treat $(u, v, h(u, v))$ as affine coordinates of $T$. Using $(U, V, W)$ as coordinate functions of planes, the dual affine equation of an LN-surface $S$ is $W=h(U, V)$. This representation says that LN-surfaces are graphs of rational functions in an affine part of dual projective space $\mathbb{P}^{3^{\star}}$.

Assume that $S$ is of class $n$, i.e. the number of tangent planes of $S$ passing through a generic line is $n$. Then the numerator $a$ and denominator $b$ of $h$ are polynomials of degrees $k$ and $l$, respectively. We change from affine coordinates $U, V, W$ to homogeneous coordinates $Y_{0}, Y_{1}, Y_{2}, Y_{3}$ by letting $U=Y_{1} / Y_{3}, V=Y_{2} / Y_{3}, W=Y_{0} / Y_{3}$. Inserting this into $W=$ $h(U, V)=a(U, V) / b(U, V)$ and multiplying with $Y_{3}^{n}$ leads to the homogeneous polynomial equation

$$
\begin{aligned}
& S: Y_{0} Y_{3}^{k-l-1} b\left(Y_{1}, Y_{2}, Y_{3}\right)-a\left(Y_{1}, Y_{2}, Y_{3}\right)=0, \text { if } k \geq l+1, \\
& S: Y_{0} b\left(Y_{1}, Y_{2}, Y_{3}\right)-Y_{3}^{l+1-k} a\left(Y_{1}, Y_{2}, Y_{3}\right)=0, \text { if } l+1 \geq k .
\end{aligned}
$$

One might call (6) the dual equation of $S$. Consider the polynomial in (6) which is sorted with respect to powers of $Y_{0}$. All partial derivatives up to order $n-2$ with respect to $Y_{0}, \ldots, Y_{3}$ vanish at $(1,0,0,0)$. This says that the plane at infinity $\omega=\mathbb{R}(1,0,0,0)$ with equation $x_{0}=0$ is an $n-1$-fold plane of $S$.

This property has the following important consequence: For any vector $\mathbf{n}=(u, v, 1)^{T}$ there exists a unique tangent plane $T(u, v)$ of $S$ having $\mathbf{n}$ as normal vector and there exists exactly one point of contact of $S$ and $T$. In other words: for any plane $E: z=a x+b y+c$ in $\mathbb{R}^{3}$ there exists exactly one tangent plane $T$ of $S$ with $E \| T$ and a unique point of contact. This unique-tangent-plane-property is the reason for the rationality of the convolution surfaces with any arbitrary rational surface which has been proved in [23]. We say that a surface $S$ satisfies the $L N$-property (4) if the tangent planes $T(u, v)$ of $S$ admit a representation (5).

We summarize these results: 
Lemma 1 The family of tangent planes $T(u, v)$ of an $L N$-surface $S$ can be represented in plane coordinates by the graph $(u, v, h(u, v))$ of a rational function $h$. The plane at infinity is an $n-1$-fold tangent plane of $S$ and this property (6) characterizes LN-surfaces. Conversely, the graph of a rational function represents the tangent planes (5) of an $L N$ surface. The convolution surfaces $S \star F$ of an $L N$-surface $S$ and any arbitrary rational surface $F$ are rational.

A particular parametric representation $\mathbf{s}(u, v)$ of a surface $S$ may not show the LN-property directly. An appropriate reparameterization may be necessary. Admissible reparameterizations are so called Cremona transformations which will be explained in Section 4.

\subsection{Quadratic triangular Bézier surfaces}

Let $\mathbf{u}=\left(u_{0}: u_{1}: u_{2}\right)$ be homogeneous coordinates in the projective plane $\mathbb{P}^{2}$. A surface $Q$ in projective 3 -space $\mathbb{P}^{3}$ admitting a parameterization of the form

$$
\mathbf{q}(\mathbf{u}) \mathbb{R}=\left(q_{0}(\mathbf{u}), \ldots, q_{3}(\mathbf{u})\right) \mathbb{R},
$$

where $q_{i}(\mathbf{u})$ are homogeneous quadratic polynomials, is called a quadratically parameterizeable surface. These so-called Steiner surfaces form a remarkable class of rational surfaces of order four and class three which have attracted the interest of mathematicians in the past $[11,15,27,28,29,30]$ and also nowadays [1, 2, 4, 5, 22, 25].

By letting $q_{0}(\mathbf{u})=u_{0}^{2}$ one obtains the family of polynomial quadratically parameterizeable surfaces. Dividing $q_{1}, q_{2}, q_{3}$ by $q_{0}$ and changing from homogeneous parameters $\left(u_{0}: u_{1}: u_{2}\right)$ to affine coordinates $(u, v)$ by $(2)$, the representation $(7)$ becomes the familiar parameterization

$$
\mathbf{s}(u, v)=\frac{1}{2} \mathbf{a} u^{2}+\mathbf{b} u v+\frac{1}{2} \mathbf{c} v^{2}+\mathbf{d} u+\mathbf{e} v+\mathbf{f} .
$$

The vectors $\mathbf{a}, \mathbf{b}, \mathbf{c}, \mathbf{d}, \mathbf{e}$, and $\mathbf{f}$ in $\mathbb{R}^{3}$ comprise the coefficients of the polynomials $q_{i}$. In general these surfaces contain a two-parameter family of parabolas (curves of degree two) corresponding to the lines in the $[u, v]$-plane. An example of this kind of surface can be seen in Fig. 1.

These surfaces are frequently called quadratic Bézier triangles and admit quadratic parameterizations in terms of barycentric coordinates with respect to a triangle in the affine parameter plane $\mathbb{R}^{2}[8]$.

\subsection{Veronese variety}

The surfaces (8) can be parameterized over the affine plane as well as over the projective plane. For the moment let $S$ be parameterized over the projective plane with coordinates 


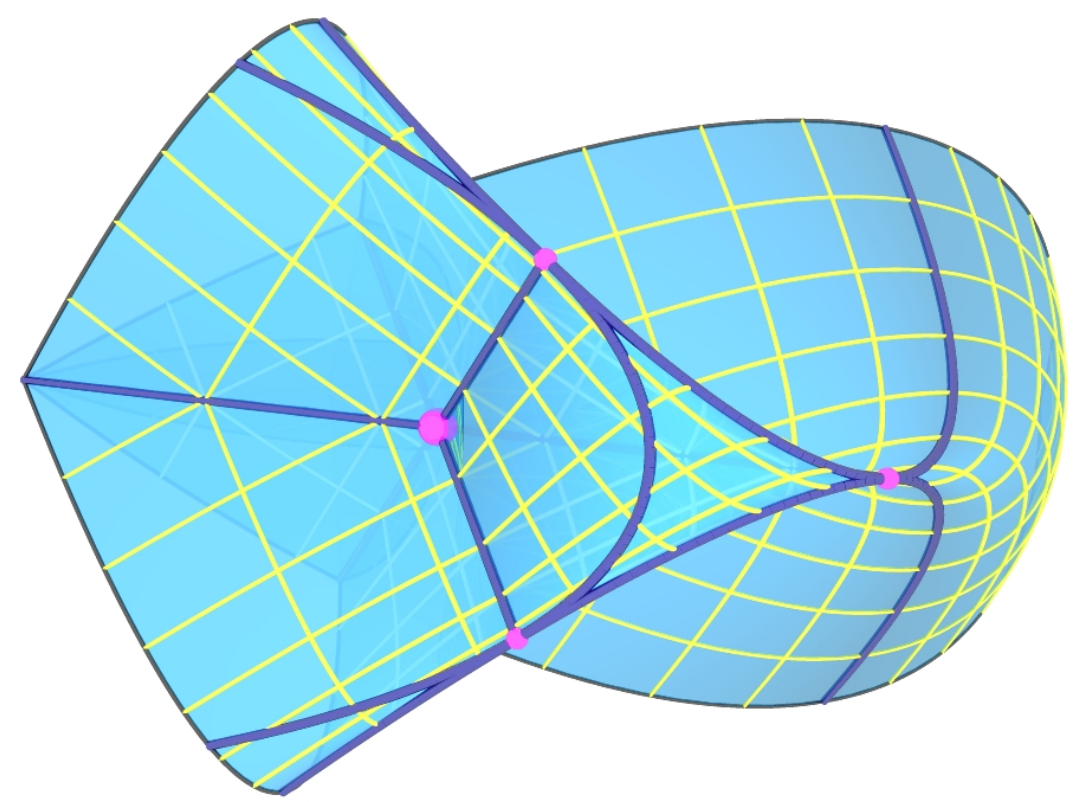

Figure 1: Quadratically parameterized Steiner surface with three double lines concurrent in the triple point and conics touching at the pinch points.

$\left(u_{0}: u_{1}: u_{2}\right)$. We consider the quadratic mapping

$$
\left(u_{0}: u_{1}: u_{2}\right) \in \mathbb{P}^{2} \mapsto\left(u_{0}^{2}: u_{0} u_{1}: u_{0} u_{2}: u_{1}^{2}: u_{1} u_{2}: u_{2}^{2}\right) \in \mathbb{P}^{5},
$$

called Veronese mapping. The right hand side of (9) is a parameterization of the Veronese variety $V_{2}^{2}$, see $[2,24]$. It is an embedding of the projective plane $\mathbb{P}^{2}$ as a regular surface in $\mathbb{P}^{5}$. It is immediately seen that the lines of $\mathbb{P}^{2}$ are mapped to the two-parameter family of conics on $V_{2}^{2}$. Any two different conics in $V_{2}^{2}$ intersect in exactly one point just like any two different lines in $\mathbb{P}^{2}$ do. As shown in $[1,5]$, all quadratically parameterizeable surfaces (7) are obtained as projections of the Veronese surface $V_{2}^{2}$.

\subsection{Projections of the Veronese variety}

We restrict ourselves to the polynomial case (8). In order to explain how to obtain the parameterization $\mathbf{s}(u, v)$ as projection of $V_{2}^{2}$, we homogenize (8) according to (2). This results in the parameterization $\mathbf{s}\left(u_{0}, u_{1}, u_{2}\right)$. The projection $\pi: \mathbb{P}^{5} \rightarrow \mathbb{P}^{3}$ which yields $\pi\left(V_{2}^{2}\right)=S$ is realized by

$$
\pi:\left[\begin{array}{c}
u_{0}^{2} \\
\mathbf{s}\left(u_{0}, u_{1}, u_{2}\right)
\end{array}\right]=\left[\begin{array}{cccccc}
1 & 0 & 0 & 0 & 0 & 0 \\
\mathbf{f} & \mathbf{d} & \mathbf{e} & \mathbf{a} & \mathbf{b} & \mathbf{c}
\end{array}\right] \cdot\left[u_{0}^{2}, u_{0} u_{1}, u_{0} u_{2}, u_{1}^{2}, u_{1} u_{2}, u_{2}^{2}\right]^{T},
$$

where $\mathbf{a}, \ldots, \mathbf{f}, \mathbf{s} \in \mathbb{R}^{3}$ are column vectors. The surfaces (10) carry a two-parameter family of conics. Some of these surfaces $(\mathbf{b}=(0,0,0))$ can be generated by translating parabolas along each other [28], others only allow more complicated kinematic generations [29]. 
Let $\omega: x_{0}=0$ be the plane at infinity in the projective extension $\mathbb{P}^{3}$ of $\mathbb{R}^{3}$ and let $c=S \cap \omega$ be the curve at infinity of $S$. From the projection (10) it follows that $c$ is obtained by $u_{0}^{2}=0$. This implies that $c$ is a double curve parameterized by

$$
\left(\mathbf{a} u_{1}^{2}+2 \mathbf{b} u_{1} u_{2}+\mathbf{c} u_{2}^{2}\right) \mathbb{R} \in \omega .
$$

Here $\left(u_{1}: u_{2}\right)$ is considered as homogeneous parameter on $c$. For linearly independent vectors $\mathbf{a}, \mathbf{b}$, and $\mathbf{c}$ one obtains conics, otherwise $c$ is degenerate.

This proves again that $\omega$ is a tangent plane of multiplicity two. Since triangular quadratic Bézier surfaces are of class three, the dual equation of $S$ looks like (6). The geometric meaning is the following: Let $g \in \mathbb{P}^{3}$ be a line in general position to $S$. Algebraically counting, there exist three tangent planes of $S$ passing through $g$. Thus for any line $h \in \omega$ in general position there is exactly one tangent plane $T \neq \omega$ passing through $h$. These synthetic geometric considerations already prove the $L N$-property of the triangular quadratic Bézier surfaces.

Corollary 2 Quadratic triangular Bézier surfaces are LN-surfaces.

Although these ideas do not lead immediately to LN-parameterizations, their existence is shown. We will present a more constructive proof of the LN-property of triangular quadratic Bézier surfaces. Therefore we investigate the normal vectors associated to the parameterization (8) and show explicitly that there exist reparameterizations of $S$ in order to obtain linearly parameterized normal vectors.

\section{Dual representation of triangular quadratic Bézier surfaces}

To prove the LN-property of a triangular quadratic Bézier surface $S$ we have to investigate the structure of the family of tangent planes. Using the affine parameterization (8) the partial derivatives of $\mathbf{s}$ with respect to $u$ and $v$ are

$$
\mathbf{s}_{, u}(u, v)=\mathbf{a} u+\mathbf{b} v+\mathbf{d}, \quad \mathbf{s}_{, v}(u, v)=\mathbf{b} u+\mathbf{c} v+\mathbf{e} .
$$

The tangent planes $T(u, v)$ of $S$ are given by

$$
T(u, v):(\mathbf{x}-\mathbf{s}(u, v))^{T} \cdot\left(\mathbf{s}_{, u} \times \mathbf{s}_{, v}\right)(u, v)=0,
$$

with support function $h=-\operatorname{det}\left(\mathbf{s}, \mathbf{s}_{, u}, \mathbf{s}_{, v}\right)$ and normal vector $\mathbf{s}_{, u} \times \mathbf{s}_{, v}$. The partial derivatives $\mathbf{s}_{, u}$ and $\mathbf{s}_{, v}$ define affine mappings in the $[u, v]$-plane. These mappings can be extended to projective mappings $p, q: \mathbb{P}^{2} \rightarrow \mathbb{P}^{2}$. Defining the matrices $P:=(\mathbf{d}, \mathbf{a}, \mathbf{b})$ and $Q:=(\mathbf{e}, \mathbf{b}, \mathbf{c})$, the projective mappings read

$$
p: \mathbf{u} \mathbb{R} \mapsto(P \mathbf{u}) \mathbb{R}, \quad q: \mathbf{u} \mathbb{R} \mapsto(Q \mathbf{u}) \mathbb{R}, \text { with } \mathbf{u}=\left(u_{0}, u_{1}, u_{2}\right)^{T} .
$$


Let $\mathbf{u}^{\star} \mathbb{R}$ be a point in $\mathbb{P}^{2}$. If rk $\left(P \mathbf{u}^{\star}, Q \mathbf{u}^{\star}\right)=1$, then $\mathbf{n}\left(\mathbf{u}^{\star}\right)=(0,0,0)^{T}$ and $\mathbf{t}\left(\mathbf{u}^{\star}\right)=$ $(0,0,0,0)^{T}$ holds. Thus we say that the parameterization $\mathbf{t}(\mathbf{u})$ of the tangent planes $T$ (or the parameterization $\mathbf{n}(\mathbf{u})$ of the normal vector) has a base point at $\mathbf{u}^{\star} \mathbb{R}$ if and only if the vectors $P \mathbf{u}^{\star}$ and $Q \mathbf{u}^{\star}$ are linearly dependent.

Excluding planar and cylindrical surfaces $S$ we can assume that the matrices $P$ and $Q$ from (13) are not scalar multiples of each other and that rk $P \geq 2$ as well as rk $Q \geq 2$. Base points allow degree reductions of parameterizations. Thus it is the key point of the reparameterization to detect the base points $\mathbf{u}^{\star} \mathbb{R}$ of the parameterization $\mathbf{t}(\mathbf{u})$ of the family of tangent planes.

\subsection{Computation of the base points}

Let $S$ be a triangular quadratic Bézier surface parameterized by (8) and let $p$ and $q$ be the projective mappings defined by (13). The computation of the base points of the dual parameterization distinguishes between the following cases, depending on the ranks of the matrices $P$ and $Q$.
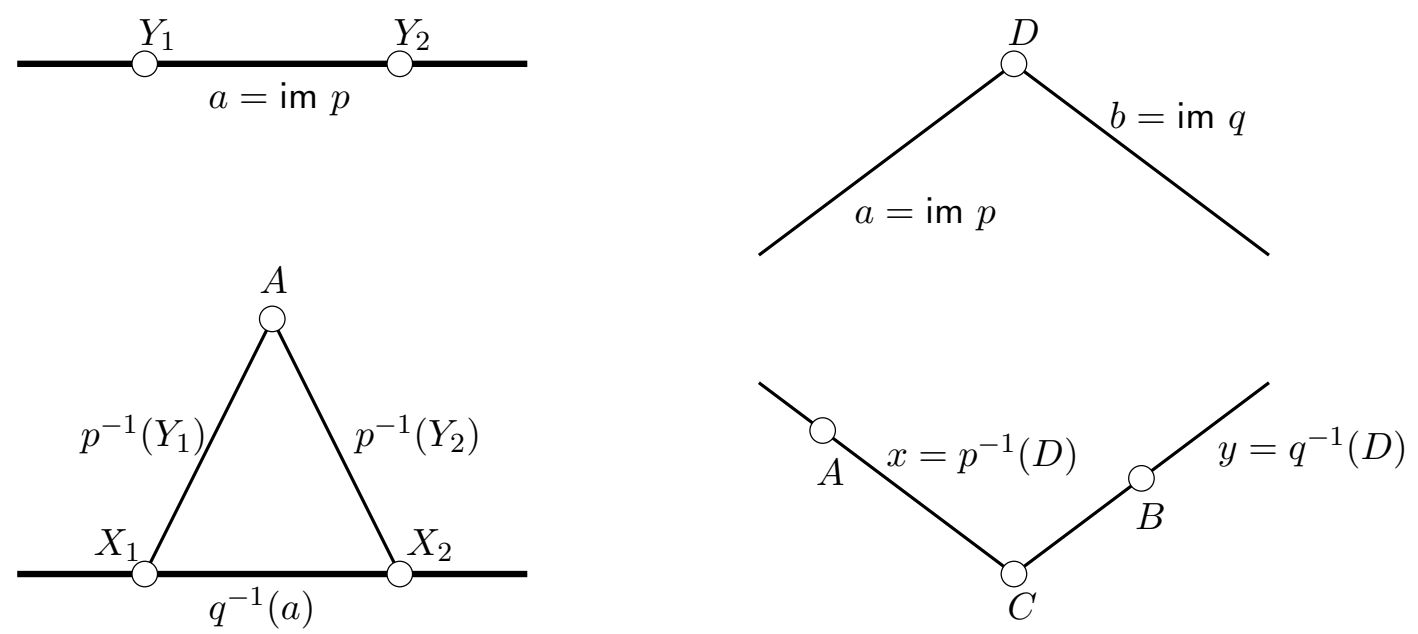

Figure 2: Construction of base points: the case rk $P=2$, rk $Q=3$ (left); the case rk $P=$ rk $Q=2$ (right).

rk $P=\mathbf{3}$ and rk $Q=\mathbf{3}$ : If both mappings $p$ and $q$ are regular, the fixed points of $q^{-1} p$ are the base points of the parameterization. Their homogeneous coordinates are the eigenvectors of the matrix $Q^{-1} P$. We obtain either three different real base points, or one real base point together with a pair of conjugate complex base points, or two real base points, or one real base point.

rk $P=2$ and rk $Q=3$ : Obviously the point $A=\mathbf{a} \mathbb{R}$ with $\mathbf{a}=\operatorname{ker} P$ is a base point. Let $a$ be the line defined by im $p \subset \mathbb{P}^{2}$ and let further be $q^{-1}(a)$ its pre-image with 
respect to $q$. The restriction of $q$ to $q^{-1}(a)$ is a projective mapping and there is either one point $X_{1}$ or there are two (real or a pair of conjugate complex) points $X_{1}$ and $X_{2}$ both contained in $q^{-1}(a)$ with $p\left(X_{i}\right)=q\left(X_{i}\right)=Y_{i}$, see Fig. 2.

Note that $q^{-1}(a)$ may pass through $A$. Finally we obtain either three real base points, or one real base point, or a pair of conjugate complex base points, two real base points, or one real base point.

If rk $P=3$ and rk $Q=2$ we interchange $u$ and $v$ in the parameterization of $\mathbf{s}$ and obtain the situation rk $P=2$ and rk $Q=3$.

rk $P=2$, rk $Q=2$ : The points $A$ and $B$ determined by ker $P$ and ker $Q$ respectively, are base points. Let $a$ and $b$ be lines defined by $\operatorname{im} p$ and $\operatorname{im} q$ and let further $D=a \cap b$, see Fig. 2. There exist fiber lines $x=p^{-1}(D)$ and $y=q^{-1}(D)$ and $C=x \cap y$ is a base point of the construction. Since $C=A$ or $C=B$ is possible and even $A=B$ might happen we obtain three, two or one real base points.

\section{Quadratic Cremona transformations}

In order to reparameterize the family of tangent planes we study quadratic Cremona transformations in the projective plane $\mathbb{P}^{2}$. Assume $\mathbf{u}=\left(u_{0}: u_{1}: u_{2}\right)$ are homogeneous coordinates of points $U$ in the projective plane $\mathbb{P}^{2}$. A mapping $\varphi: \mathbb{P}^{2} \rightarrow \mathbb{P}^{2}$ with $U \mapsto V$ is called a quadratic Cremona transformation if the homogeneous coordinates $\mathbf{v} \mathbb{R}$ of the image points $V$ can be expressed in the form

$$
\mathbf{v} \mathbb{R}=\left(q_{0}(\mathbf{u}): q_{1}(\mathbf{u}): q_{2}(\mathbf{u})\right)
$$

where $q_{i}$ are homogeneous quadratic polynomials and additionally the inverse $\varphi^{-1}$ is of the same form. Because of the latter property Cremona transformations are often called birational.

Here we remark that $q_{i}$ cannot be prescribed independently. They have to satisfy certain relations in order to define a birational transformation, see [7]. It is obvious that $q_{i}$ are the equations of conics in $\mathbb{P}^{2}$. From the theory of Cremona transformations we know that these three conics have to have three points in common. These points are called fundamental points of the transformation and define their exceptional set. Sometimes it occurs that two or all three of the base points coincide. This gives rise to a projective classification of quadratic planar Cremona transformations.

In the following we give a brief overview on certain sets of conics, namely pencils and nets, in the projective plane in order to understand the geometry behind Cremona transformations. 


\subsection{Conics}

It is well known that a conic $k$ (or more generally speaking: a curve of degree two) can be defined as the set of points $X$ with homogeneous coordinate vectors $\mathbf{x} \mathbb{R}=\left(x_{0}, x_{1}, x_{2}\right) \mathbb{R}$ satisfying a homogeneous quadratic polynomial equation

$$
k: \mathbf{x}^{T} K \mathbf{x}=0
$$

where $K$ is a symmetric $3 \times 3$-matrix with real entries. In order to avoid lengthy discussions we view those subsets of $\mathbb{P}^{2}$ defined by singular matrices $K$ also as conics and call them singular. This includes pairs of lines (real ones or a pair of conjugate complex ones) or a single double line, depending on the normal form of $K$. If rk $K=3$ and $K$ is positive definite the curve $k$ is empty (in the real projective plane).

\subsection{Pencils of conics}

Let $k$ and $l$ be two curves of degree two given by the respective equations $k: \mathbf{x}^{T} K \mathbf{x}=0$ and $l: \mathbf{x}^{T} L \mathbf{x}=0$. The curves are assumed to be distinct which is guaranteed if $K \neq \lambda L$. We call the set $\mathcal{P}$ of conics (including singular ones) defined by the linear combination

$$
\mathcal{P}: \mathbf{x}^{T}(\kappa K+\lambda L) \mathbf{x}=0
$$

a pencil of conics. The pencil $\mathcal{P}$ can be spanned by any two different conics in it. The singular conics contained in $\mathcal{P}$ which correspond to the solutions $(\kappa: \lambda)$ of the homogeneous cubic equation $\operatorname{det}(\kappa K+\lambda L)=0$ can also serve as base conics.

There are five types of pencils of conics depending on the number of common points and/or common tangents of the conics in the pencil. Note that we do not care whether an intersection point of two conics is a real or a complex one.
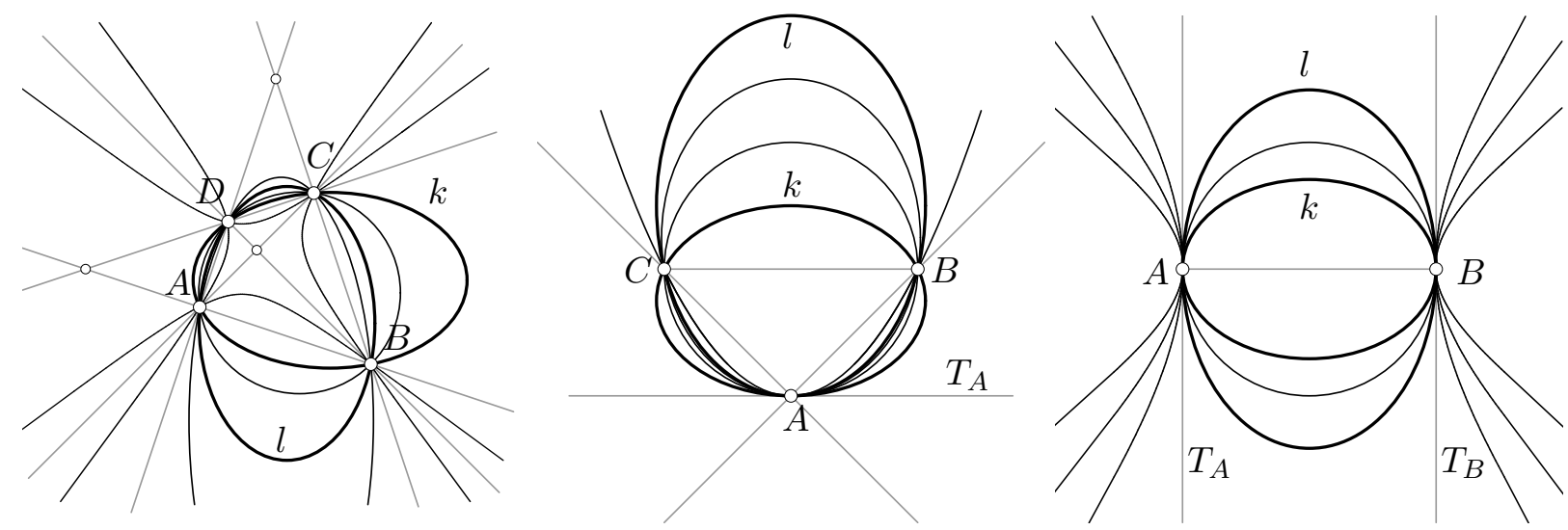

Figure 3: Pencils of conics 1: first, second, and third kind. 
Four common points: Two conics $k$ and $l$ and thus all conics in the pencil share the points $A, B, C$, and $D$. These points form a quadrangle by obvious reasons. The pencil $\mathcal{P}$ contains three different singular conics, i.e. three pairs of lines $(A B, C D)$, $(A C, B D),(A D, B C)$, see Fig. 3.

Two points and one line element: ${ }^{1}$ Any two different conics $k$ and $l$ in $\mathcal{P}$ intersect at the points $A, B$, and $C$ while touching at $A$ the common tangent $T_{A}$. This pencil contains two singular conics: the pairs of lines $\left(B C, T_{A}\right)$ and $(A B, A C)$, see Fig. 3.

Two line elements: Any two conics $k$ and $l$ in $\mathcal{P}$ share the line elements $\left(A, T_{A}\right)$ and $\left(B, T_{B}\right)$, i.e. any two conics of $\mathcal{P}$ touch at $A$ the line $T_{A}$, analogoulsy at $B$ the line $T_{B}$. The singular conics in this pencil are the double line $A B$ and the pair $\left(T_{A}, T_{B}\right)$ of lines, see Fig. 3.

Point and osculating element: Any two conics of the pencil are in contact of order two at the point $A$. Thus they share the line element $\left(A, T_{A}\right)$ and a further point $B \neq A$. The pair $\left(A B, T_{A}\right)$ of lines is the only singular conic in the pencil, see Fig. 4.

When viewing $A$ as the intersection of two conics $k$ and $l$ in $\mathcal{P}$, it is of multiplicity three. The conics $k$ and $l$ are said to osculate each other at $A$.

Hyperosculating element: Any two conics in the pencil are in contact of order three at the line element $\left(A, T_{A}\right)$. The only singular conic in the pencil is the double line $T_{A}$, see Fig. 4.

Considered as a point of intersection of any two conics in this pencil, the point $A$ is of multiplicity four. Any two conics in the pencil are said to hyperosculate each other at $A$.

We call a pencil of conics of the first, second, third, fourth, or fifth kind according to its place in our list.

\subsection{Nets of conics}

By removing one condition (one point) from the determining elements of a pencil of conics one obtains a net of conics. Note that this is a special kind of a two-parameter family of conics: conics passing through three points, or conics sharing a line element and a further point, or conics osculating each other at a certain point.

For any quadratic Cremona transformation $\varphi: \mathbb{P}^{2} \rightarrow \mathbb{P}^{2}$ there exists an associated net of conics. The fundamental set of the net are the base points of $\varphi$, where all the conics of the net are passing through.

\footnotetext{
${ }^{1}$ We call the pair consisting of a point $P$ and an incident line $L$ a line element and denote it by $(P, L)$.
} 

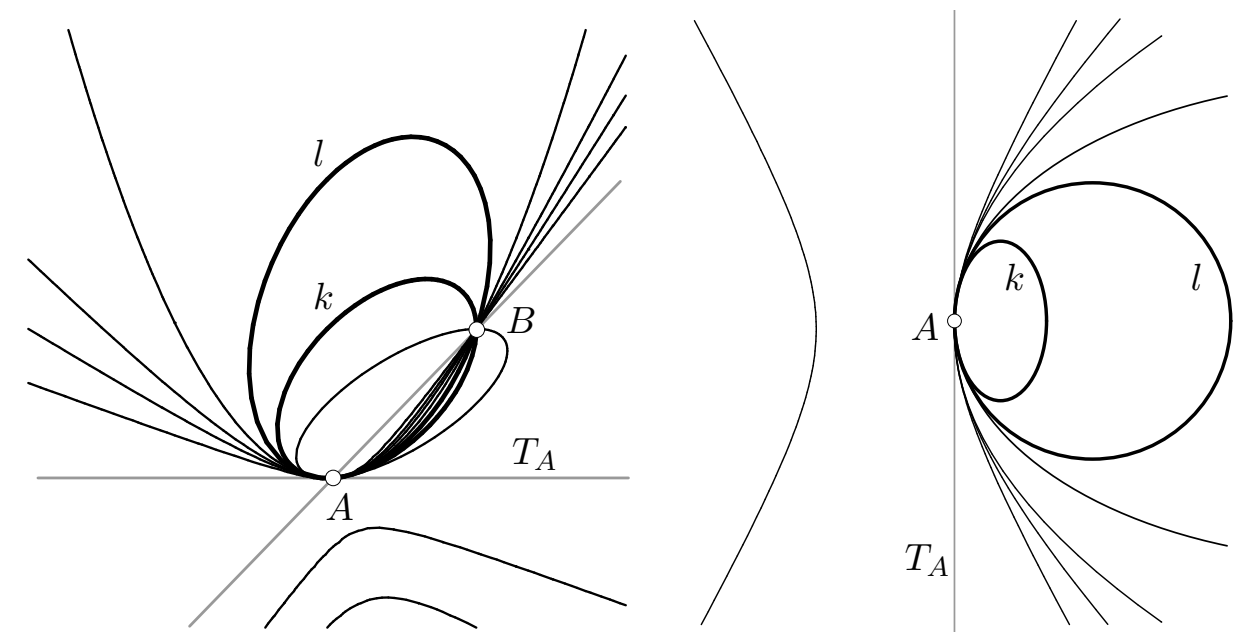

Figure 4: Pencils of conics 2: fourth and fifth kind

Conics through three points: We let $A=(1: 0: 0), B=(0: 1: 0)$ and $C=(0: 0: 1)$ be the three base points of the net of conics. It is spanned by the three pairs of lines $x_{1} x_{2}=0, x_{2} x_{0}=0$, and $x_{0} x_{1}=0$. Thus the general conic $c$ of the net has the equation $\alpha x_{1} x_{2}+\beta x_{2} x_{0}+\gamma x_{0} x_{1}=0$, where $(\alpha: \beta: \gamma) \neq(0: 0: 0)$. The Cremona transformation $\varphi$ with this fundamental set and its inverse are given by

$$
\begin{aligned}
\varphi: & \left(x_{0}: x_{1}: x_{2}\right) \mapsto\left(x_{0}^{\prime}: x_{1}^{\prime}: x_{2}^{\prime}\right)=\left(x_{1} x_{2}: x_{0} x_{2}: x_{0} x_{1}\right), \\
\varphi^{-1}: \quad & \left(x_{0}^{\prime}: x_{1}^{\prime}: x_{2}^{\prime}\right) \mapsto\left(x_{0}: x_{1}: x_{2}\right)=\left(x_{1}^{\prime} x_{2}^{\prime}: x_{0}^{\prime} x_{2}^{\prime}: x_{0}^{\prime} x_{1}^{\prime}\right) .
\end{aligned}
$$

The net of conics appearing here is obtained from a pencil of the first kind by removing one base point.

Conics through one point and one line element: We choose the line element $A=$ $(1: 0: 0)$ and $a: x_{2}=0$ and the additional point $B=(0: 0: 1)$. The net is spanned by the curves $x_{1} x_{2}=0, x_{0} x_{2}=0$, and $x_{1}^{2}=0$. The generic equation of a curve of the net is $\alpha x_{1} x_{2}+\beta x_{2} x_{0}+\gamma x_{1}^{2}=0$, where $(\alpha: \beta: \gamma) \neq(0: 0: 0)$. The thus defined Cremona transformation and its inverse are given by

$$
\begin{aligned}
\varphi: & \left(x_{0}: x_{1}: x_{2}\right) \mapsto\left(x_{0}^{\prime}: x_{1}^{\prime}: x_{2}^{\prime}\right)=\left(x_{1} x_{2}: x_{0} x_{2}: x_{1}^{2}\right), \\
\varphi^{-1}: & \left(x_{0}^{\prime}: x_{1}^{\prime}: x_{2}^{\prime}\right) \mapsto\left(x_{0}: x_{1}: x_{2}\right)=\left(x_{1}^{\prime} x_{2}^{\prime}: x_{0}^{\prime} x_{2}^{\prime}: x_{0}^{\prime 2}\right)
\end{aligned}
$$

The net of conics associated with this type of Cremona transformation is obtained from a pencil of the second kind by removing one base point different from the point of contact.

Osculating element: Consider the conic $k: x_{1}^{2}-x_{0} x_{2}=0$ and the point $A=(1: 0: 0)$. The net is formed by all curves $c$ of degree two osculating $k$ at $A$. It is spanned by $k$ and the pair of lines with equations $x_{1} x_{2}=0$ and $x_{2}^{2}=0$. The Cremona 
transformation and its inverse are given by

$$
\begin{aligned}
\varphi: & \left(x_{0}: x_{1}: x_{2}\right) \mapsto\left(x_{0}^{\prime}: x_{1}^{\prime}: x_{2}^{\prime}\right)=\left(x_{1} x_{2}: x_{1}^{2}-x_{0} x_{2}: x_{2}^{2}\right), \\
\varphi^{-1}: & \left(x_{0}^{\prime}: x_{1}^{\prime}: x_{2}^{\prime}\right) \mapsto\left(x_{0}: x_{1}: x_{2}\right)=\left(x_{0}^{\prime 2}-x_{1}^{\prime} x_{2}^{\prime}: x_{0}^{\prime} x_{2}^{\prime}: x_{2}^{\prime 2}\right) .
\end{aligned}
$$

The associated net of conics is obtained from the pencil of conics of the fourth kind by removing the base point different from the point of osculation.

The pencils of third and fifth kind do not lead to base conics of quadratic Cremona transformations. Removing one base point removes all of them in case of a pencil of fifth kind. It leaves only two base points or a line element in the case of a pencil of third kind and the set of conics through this elements is a three-parametric one.

A Cremona transformation $\varphi$ maps a line $g$ in general position to a conic $\varphi(g)$ passing through the base points. Any conic $c$ in general position is mapped to a rational curve $\varphi(c)$ of degree four. The conics of the associated net pass through the base points and are thus mapped to straight lines. This will help to reparameterize the tangent planes of a quadratically parameterizeable surface when proving the LN-property.

\section{Proving the LN-property}

Given a quadratic triangular Bézier surface $S$ with polynomial parameterization (8) we show that $S$ satisfies the LN-property. This means that the tangent planes $T(u, v)$ admit a representation (5). The proof is splitted into two parts: At first let $\mathbf{n}=\mathbf{s}_{, u} \times \mathbf{s}_{v}$ be a normal vector field of $\mathbf{s}$. We show that the conics $\mathbf{n}_{i}(u, v)=0$ determined by $\mathbf{n}$ 's coordinate functions define a net of conics. Second we give a practical reparameterization method which is illustrated at hand of examples in Sec. 6.

Theorem 3 The conics $\mathbf{n}_{i}(u, v)=0, i=1,2,3$ form a net.

Proof: Assume $S$ is parameterized by $\mathbf{s}$ from (8). The normal vector $\mathbf{n}(u, v)$ reads

$$
\begin{gathered}
\mathbf{n}(u, v)=(\mathbf{a} \times \mathbf{b}) u^{2}+(\mathbf{a} \times \mathbf{c}) u v+(\mathbf{b} \times \mathbf{c}) v^{2} \\
+(\mathbf{a} \times \mathbf{e}+\mathbf{d} \times \mathbf{b}) u+(\mathbf{b} \times \mathbf{e}+\mathbf{d} \times \mathbf{c}) v+\mathbf{d} \times \mathbf{e} .
\end{gathered}
$$

The coordinate functions of $\mathbf{n}$ define conics $\mathbf{n}_{i}(u, v)=0$ in the $[u, v]$-plane. We show that these conics form a net. Consider two conics $\mathbf{n}_{1}(u, v)=0$ and $\mathbf{n}_{2}(u, v)=0$. The $u$-coordinates of their intersection points are the zeros of the resultant $\operatorname{Res}\left(\mathbf{n}_{1}, \mathbf{n}_{2}, v\right)$ of $\mathbf{n}_{1}$ and $\mathbf{n}_{2}$ with respect to $v$. We prove that the resultants $\operatorname{Res}\left(\mathbf{n}_{1}, \mathbf{n}_{2}, v\right), \operatorname{Res}\left(\mathbf{n}_{2}, \mathbf{n}_{3}, v\right)$, and 
$\operatorname{Res}\left(\mathbf{n}_{1}, \mathbf{n}_{3}, v\right)$ with respect to $v$ share a cubic factor $p(u)$ and thus there are three common points (algebraically counted) of the conics $\mathbf{n}_{i}=0$. The resultants are

$$
\begin{aligned}
& \operatorname{Res}\left(\mathbf{n}_{1}, \mathbf{n}_{2}, v\right)=\left(b_{3} e_{3}-c_{3} d_{3}+\left(b_{3}^{2}-a_{3} c_{3}\right) u\right) p(u), \\
& \operatorname{Res}\left(\mathbf{n}_{2}, \mathbf{n}_{3}, v\right)=\left(b_{1} e_{1}-c_{1} d_{1}+\left(b_{1}^{2}-a_{1} c_{1}\right) u\right) p(u), \\
& \operatorname{Res}\left(\mathbf{n}_{1}, \mathbf{n}_{3}, v\right)=\left(b_{2} e_{2}-d_{2} c_{2}+\left(b_{2}^{2}-a_{2} c_{2}\right) u\right) p(u) .
\end{aligned}
$$

The zeros of $p(u)$ determine the $u$-coordinates of three intersection points of any pair of the conics $\mathbf{n}_{i}(u, v)=0$. The number and the multiplicity of intersection points in independent of the chosen coordinate system. This proves that the conics $\mathbf{n}_{i}(u, v)=0$ share three points (algebraically counted) which are the base points of the corresponding Cremona transformation. The coefficients of the polynomial $p(u)=\sum_{i} c_{i} u^{i}$ are listed below.

In addendum we give the coefficients of the cubic polynomial $p(u)$ :

$$
\begin{aligned}
c_{3}= & \operatorname{det}(\mathbf{a}, \mathbf{b}, \mathbf{c})^{2}, \\
c_{2}= & \operatorname{det}(\mathbf{a}, \mathbf{b}, \mathbf{c})(2 \operatorname{det}(\mathbf{b}, \mathbf{c}, \mathbf{d})-\operatorname{det}(\mathbf{a}, \mathbf{c}, \mathbf{e})) \\
c_{1}= & 2 \operatorname{det}(\mathbf{a}, \mathbf{b}, \mathbf{c}) \operatorname{det}(\mathbf{c}, \mathbf{d}, \mathbf{e})+\operatorname{det}(\mathbf{a}, \mathbf{b}, \mathbf{e}) \operatorname{det}(\mathbf{b}, \mathbf{c}, \mathbf{e}) \\
& +\operatorname{det}(\mathbf{b}, \mathbf{c}, \mathbf{d})^{2}-\operatorname{det}(\mathbf{a}, \mathbf{c}, \mathbf{d}) \operatorname{det}(\mathbf{b}, \mathbf{c}, \mathbf{e}) \\
c_{0}= & \operatorname{det}(\mathbf{b}, \mathbf{c}, \mathbf{d}) \operatorname{det}(\mathbf{c}, \mathbf{d}, \mathbf{e})-\operatorname{det}(\mathbf{b}, \mathbf{c}, \mathbf{e}) \operatorname{det}(\mathbf{b}, \mathbf{d}, \mathbf{e})
\end{aligned}
$$

Theorem 4 The quadratic triangular Bézier surfaces are LN surfaces.

Proof: Assume that $S$ is given by the parameterization (8). We have to prove that the tangent planes of $S$ admit the representation (5). The partial derivatives $\mathbf{s}_{, u}$ and $\mathbf{s}_{, v}$ according to (11) define projective mappings $p$ and $q$ given by (13). The base points of the parameterization $\mathbf{n}(u, v)$ of the normal vector field are uniquely determined by $p$ and $q$ and computed according to Sec. 3.1. The configuration of the base points defines the type of net of conics determined by $\mathbf{n}_{i}(u, v)=0$.

Depending on the type of net of conics one can perform a coordinate transformation in order to apply the normal form of the appropriate Cremona transformation from Sec. 4.3 This transforms the net of conics to the two-parameter family of straight lines of $\mathbb{P}^{2}$. Thus the normals of $S$ become linearly parameterized and this completes the proof of the LNproperty.

It would have been sufficient to prove the LN-property for the affine normal forms given in [22]. The parameterizations $\left(u^{2}+v^{2}, u, v\right)$ and $(u v, u, v)$ describe paraboloids, further $\left(u^{2}, u, v\right),\left(u^{2}, v^{2}, u\right)$, and $\left(u^{2}, u v, u\right)$ are patches on parabolic cylinders and finally 
$\left(u^{2}, v^{2}, u v\right)$ is a cone. Since the LN-property of these quadrics is obvious we focus on the nine remaining surface classes of degree three and four. Table 1 shows the nine types of affine normal forms of quadratic triangular Bézier surfaces of degree $>2$ according to [22]. We list the parameterization, the type of Cremona transformation, the affine form of the reparameterization, and finally the homogeneous equation in dual coordinates proving that these surfaces possess $\omega: x_{0}=0$ as tangent plane of multiplicity two, compare Sec. 2.4. Line three and four of table 1 represent two ruled surfaces of order three: The Whitney umbrella, an affine version of Plücker's conoid and the Cayley surface, respectively.

Table 1: Affine normal forms, type of Cremona transformation and dual equation of quadratic triangular Bézier surfaces.

\begin{tabular}{|l|l|l|l|l|}
\hline & Parameterization & Type & Transformation & Dual homogeneous equation \\
\hline \hline 1 & $\mathbf{f}=\left(u^{2}, v^{2}, u+v\right)$ & 1 & $u=\frac{-1}{2 s}, v=\frac{-1}{2 t}$ & $4 Y_{0} Y_{1} Y_{2}-Y_{3}^{2}\left(Y_{1}+Y_{2}\right)=0$ \\
\hline 2 & $\mathbf{f}=\left(u^{2}, v^{2}+u, v\right)$ & 2 & $u=\frac{-t}{2 s}, v=\frac{-1}{2 t}$ & $4 Y_{0} Y_{1} Y_{2}-Y_{2}^{3}-Y_{1} Y_{3}^{2}=0$ \\
\hline 3 & $\mathbf{f}=\left(u^{2}, u v, v\right)$ & 2 & $u=\frac{-1}{t}, v=\frac{2 s}{t^{2}}$ & $Y_{0} Y_{2}^{2}+Y_{1} Y_{3}^{2}=0$ \\
\hline 4 & $\mathbf{f}=\left(u^{2}+v, u v, u\right)$ & 3 & $u=\frac{-s}{t}, v=\frac{2 s^{2}-t}{t^{2}}$ & $Y_{0} Y_{2}^{2}+Y_{1}^{3}-Y_{1} Y_{2} Y_{3}=0$ \\
\hline 5 & $\mathbf{f}=\left(u^{2}-v^{2}, u v, u\right)$ & 1 & $u=\frac{-2 s}{4 s^{2}+t^{2}}, v=\frac{-t}{4 s^{2}+t^{2}}$ & $Y_{0}\left(Y_{2}^{2}+4 Y_{1}^{2}\right)-Y_{1} Y_{3}^{2}=0$ \\
\hline 6 & $\mathbf{f}=\left(u^{2}, v^{2}, u v+u\right)$ & 2 & $u=\frac{2 t}{1-4 s t}, v=\frac{-1}{1-4 s t}$ & $Y_{0}\left(4 Y_{1} Y_{2}-Y_{3}^{2}\right)-Y_{2} Y_{3}^{2}=0$ \\
\hline 7 & $\mathbf{f}=\left(u^{2}, v^{2}, u v+u+v\right)$ & 1 & $u=\frac{2 t-1}{1-4 s t}, v=\frac{2 s-1}{1-4 s t}$ & $\begin{array}{l}Y_{0}\left(4 Y_{1} Y_{2}-Y_{3}^{2}\right)+Y_{3}^{3} \\
-Y_{3}^{2}\left(Y_{1}+Y_{2}\right)=0\end{array}$ \\
\hline 8 & $\mathbf{f}=\left(u^{2}, v^{2}+u, u v\right)$ & 3 & $u=\frac{2 t^{2}}{1-4 s t}, v=\frac{-t}{1-4 s t}$ & $Y_{0}\left(4 Y_{1} Y_{2}-Y_{3}^{2}\right)+Y_{2}^{3}=0$ \\
\hline 9 & $\mathbf{f}=\left(u^{2}, v^{2}+u, u v-v\right)$ & 2 & $u=\frac{1+2 t^{2}}{1-4 s t}, v=\frac{-t-2 s}{1-4 s t}$ & $\begin{array}{l}Y_{0}\left(4 Y_{1} Y_{2}-Y_{3}^{2}\right)-Y_{2}^{3} \\
-Y_{3}^{2}\left(Y_{1}+Y_{2}\right)=0\end{array}$ \\
\hline
\end{tabular}

\section{$6 \quad$ Examples}

In this section we demonstrate the reparameterization method at hand of three examples of the affine normal forms given in [22] corresponding to different types of Cremona transformations.

1. Let $\mathbf{s}=\left(u^{2}, v^{2}, u+v\right)^{T}$, which is the the affine normal form 1 from table 1 . An example is shown in Fig. 5. This surface can be obtained by translating the parabolas $\left(u^{2}, 0, u\right)^{T}$ and $\left(0, v^{2}, v\right)^{T}$ along each other. It is one of the surfaces mentioned in [28]. We find the normal vector $\mathbf{n}=(-2 v,-2 u, 4 u v)^{T}$, or (depending on homogeneous parameters $) \mathbf{n}=\left(-2 u_{0} u_{2},-2 u_{0} u_{1}, 4 u_{1} u_{2}\right)^{T}$. The associated projective mappings $p$ 

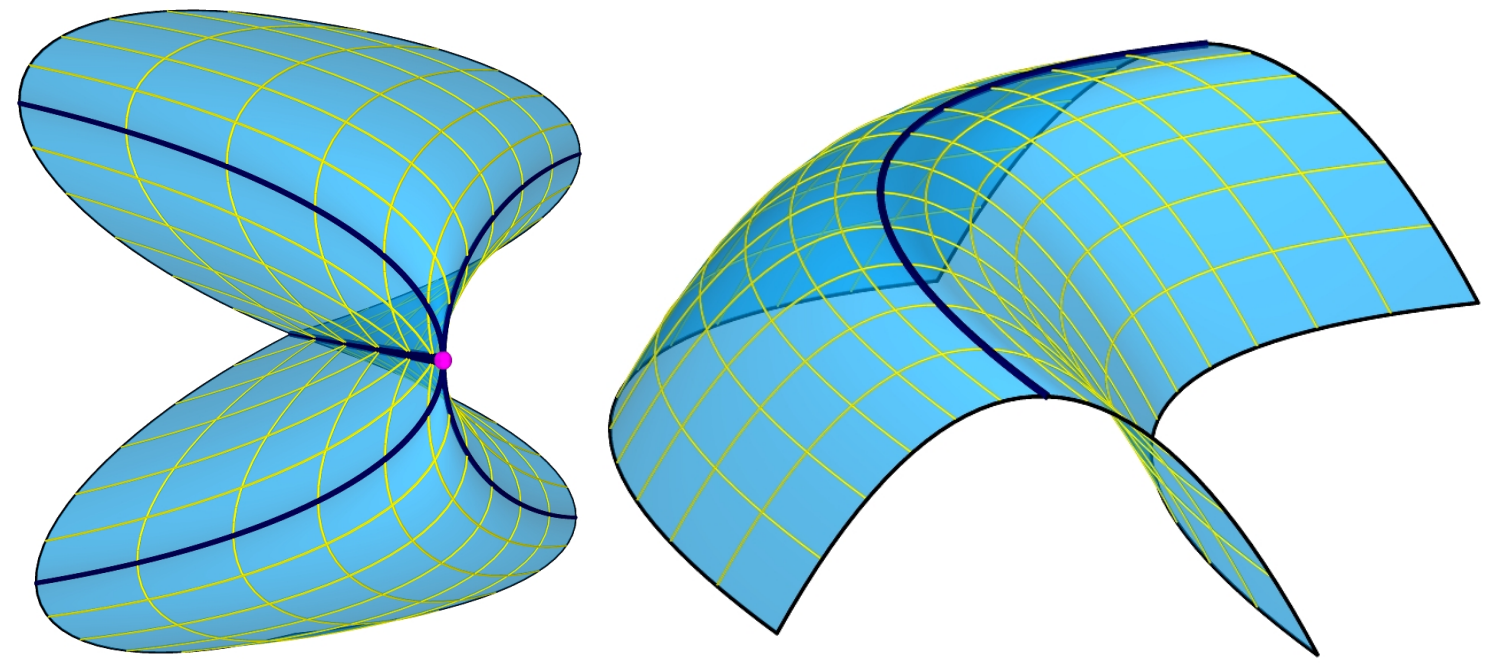

Figure 5: Quadratically parameterized surfaces: Affine normal forms 1 and 2.

and $q$ from (13) are both singular: rk $P=$ rk $Q=2$. The corresponding Cremona transformation is of type 1 and reads

$$
\varphi^{-1}: u_{0}=2 u_{1}^{\prime} u_{2}^{\prime}, \quad u_{1}=-u_{0}^{\prime} u_{2}^{\prime}, \quad u_{2}=-u_{0}^{\prime} u_{1}^{\prime} .
$$

With this reparameterization the normal $\mathbf{n}$ changes to $\left(u_{1}^{\prime}, u_{2}^{\prime}, u_{0}^{\prime}\right)^{T}$ after cancellation of the factor $4 u_{0}^{\prime} u_{1}^{\prime} u_{2}^{\prime}$. In terms of affine coordinates $u$ and $v$ the Cremona transformation $\varphi$ is

$$
\varphi^{-1}: u=-\frac{1}{2 s}, \quad v=-\frac{1}{2 t}
$$

and yields the affine parameterization of the normal vector $\mathbf{n}=(s, t, 1)^{T}$. Here and in the following we let $u_{1}^{\prime} / u_{0}^{\prime}=s$ and $u_{2}^{\prime} / u_{0}^{\prime}=t$. This leads to the LN-parameterization s and the representation of $S$ 's tangent planes by

$$
\mathbf{s}(s, t)=\frac{1}{4 s^{2} t^{2}}\left(t^{2}, s^{2},-2 s t(s+t)\right)^{T}, \quad T(s, t): \frac{s+t}{4 s t}+s x+t y+z=0 .
$$

So we find the dual equation of the surface $S$ as $4 Y_{0} Y_{1} Y_{2}-Y_{3}^{2}\left(Y_{1}+Y_{2}\right)=0$.

2. Let $S$ be parameterized by $\mathbf{s}=\left(u^{2}, v^{2}+u, v\right)^{T}$, affine normal form 2 from table 1 , which can be obtained by translating the parabolas $\left(u^{2}, u, 0\right)^{T}$ and $\left(0, v^{2}, v\right)^{T}$ along each other, see [28]. An example is shown in Fig. 5. Its normal vector is $\mathbf{n}=$ $(1,-2 u, 4 u v)^{T}$ and the homogeneous version is $\left(u_{0}^{2},-2 u_{0} u_{1}, 4 u_{1} u_{2}\right)^{T}$. The base points are given by ker $Q$ and ker $P$, respectively. Since there exists no additional base point, the suitable Cremona transformation is of type 2 and reads

$$
\varphi^{-1}: u_{0}=-4 u_{1}^{\prime} u_{2}^{\prime}, \quad u_{1}=2 u_{2}^{\prime 2}, \quad u_{2}=2 u_{0}^{\prime} u_{1}^{\prime} .
$$


The normal changes to $\left(u_{1}^{\prime}, u_{2}^{\prime}, u_{0}^{\prime}\right)^{T}$ if we remove the dispensable factor $16 u_{2}^{\prime 2} u_{1}^{\prime}$. In terms of affine parameters $\varphi$ reads

$$
\varphi^{-1}: u=-\frac{t}{2 s}, \quad v=-\frac{1}{2 t} .
$$

and yields the affine LN-parameterization $\mathbf{s}$ of $S$ and its tangent planes $T$

$$
\mathbf{s}(s, t)=\frac{1}{4 s^{2} t^{2}}\left(t^{4}, s\left(s-2 t^{3}\right),-2 s^{2} t\right)^{T}, \quad T(s, t): \frac{s+t^{3}}{4 s t}+s x+t y+z=0 .
$$

The dual equation of the surface reads $4 Y_{0} Y_{1} Y_{2}-Y_{2}^{3}-Y_{3}^{2} Y_{1}=0$.

3. Let $S$ be parameterized by $\mathbf{s}=\left(u^{2}, v^{2}+u, u v\right)^{T}$ (affine normal form 8). An example is displayed in Fig. 6. Its normal vector is either $\mathbf{n}=\left(u-2 v^{2},-2 u^{2}, 4 u v\right)^{T}$ or $\left(u_{0} u_{1}-2 u_{2}^{2},-2 u_{1}^{2}, 4 u_{1} u_{2}\right)^{T}$. There is only one base point determined by ker $Q$ and the suitable Cremona transformation of type 3 is

$$
\varphi^{-1}: u_{0}=u_{0}^{\prime 2}-4 u_{1}^{\prime} u_{2}^{\prime}, \quad u_{1}=2 u_{2}^{\prime 2}, \quad u_{2}=-u_{1}^{\prime} u_{2}^{\prime} .
$$

An affine version of $\varphi$ is given by

$$
\varphi^{-1}: u=\frac{2 t^{2}}{1-4 s t}, \quad v=\frac{-t}{1-4 s t} .
$$

The LN-parameterization of $S$ and the equation of its tangent planes are

$\mathbf{s}(s, t)=\frac{1}{(4 s t-1)^{2}}\left(4 t^{4},-t^{2}(8 s t-3),-2 t^{3}\right)^{T}, \quad T(s, t): \frac{s+t-1}{4 s t-1}+s x+t y+z=0$.

Thus the dual equation of $S$ reads $Y_{0}\left(4 Y_{1} Y_{2}-Y_{3}^{2}\right)-Y_{2}^{3}=0$.

\section{Convolution surface}

Finally we compute the convolution surface $C=F \star G$ of two quadratically polynomial parameterized surfaces.

- Let $F$ and $G$ be given by $\mathbf{f}=\left(u^{2}, v^{2}+u, v\right)^{T}$ and $\mathbf{g}=\left(s t, s^{2}, s+t\right)^{T}$, respectively. First we reparameterize both surfaces $F$ and $G$ such that equal parameters correspond to parallel normals. With the normals $\mathbf{n}_{F}=(1,-2 u, 4 u v)^{T}$ and $\mathbf{n}_{G}=\left(2 s, s-t,-2 s^{2}\right)^{T}$ of $F$ and $G$ we find the affine versions of the Cremona transformations in the $[u, v]$ plane and in the $[s, t]$-plane, respectively:

$$
\varphi_{F}^{-1}: u=-\frac{y}{2 x}, \quad v=-\frac{1}{2 y}, \quad \varphi_{G}^{-1}: s=-\frac{1}{x}, \quad t=\frac{2 y-x}{x^{2}} .
$$



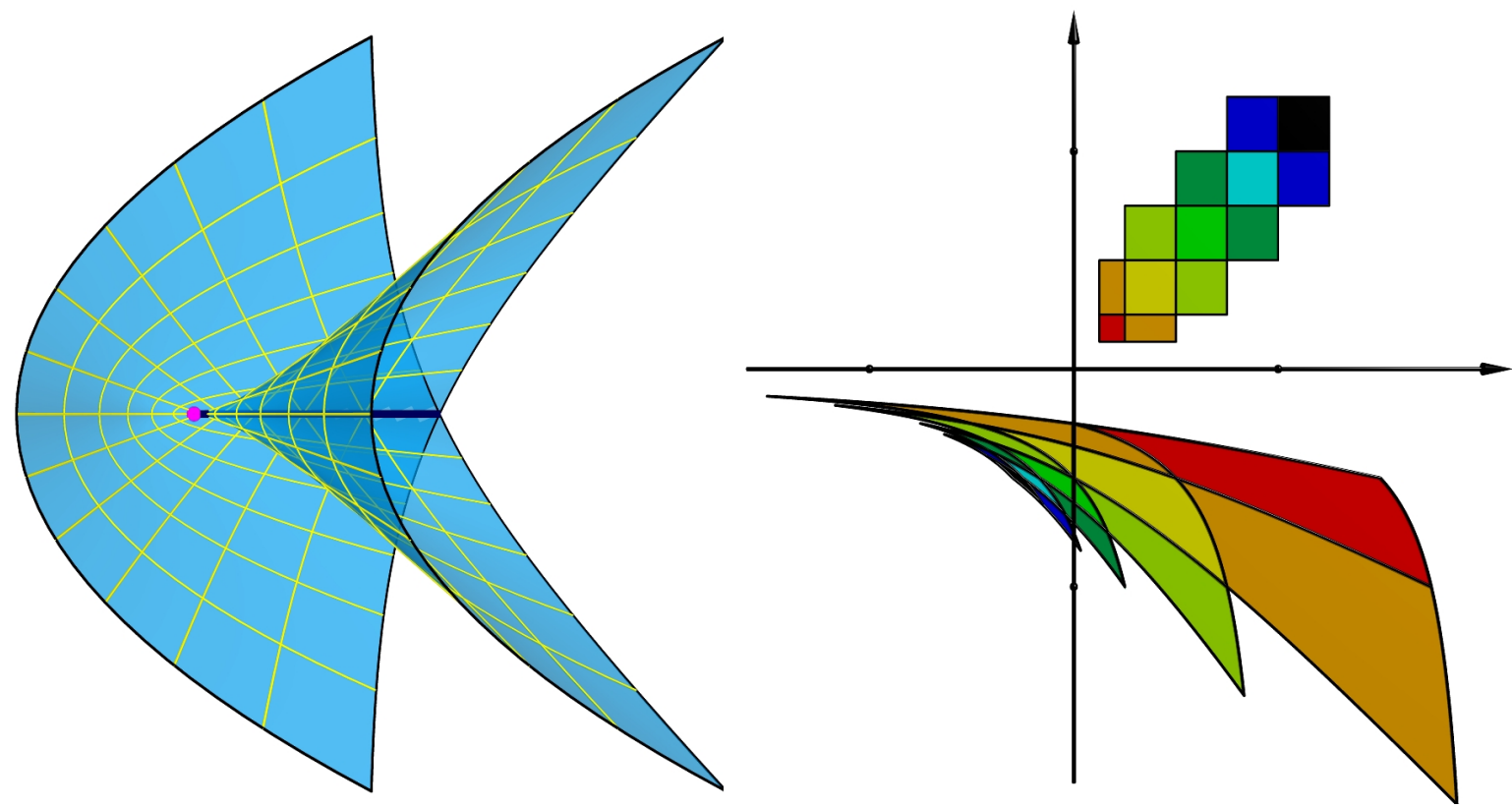

Figure 6: Quadratically parameterized surface: Affine normal form 8 (left) and the straightening of the parameter lines via the Cremona transformation from Equ. (26) (right).

Inserting the latter equations into the parameterizations of $F$ and $G$ we obtain

$$
\mathbf{f}(x, y)=\frac{1}{4 x^{2} y^{2}}\left(y^{4}, x\left(x-2 y^{3}\right),-2 x^{2} y\right)^{T}, \quad \mathbf{g}(x, y)=\frac{1}{x^{3}}(x-2 y, x, 2 x(y-x))^{T} .
$$

A parameterization $\mathbf{c}$ of the convolution surface $C$ is thus computed as the sum of $\mathbf{f}(x, y)$ and $\mathbf{g}(x, y)$ and thus we have

$$
\mathbf{c}(x, y)=\frac{1}{4 x^{2} y^{2}}\left(x y^{2}+4 x-8 y, x^{2}-2 x y^{3}+4 y^{2}, 4 y^{2}-x^{2}-4 x y\right)^{T} .
$$

Fig. 7 shows the surfaces $F$ and $G$ as well as their convolution surface $C$.

- Let $F$ and $G$ be given by $\mathbf{f}=\left(2 u^{2}, 2 v^{2}, 2(u+v)\right)^{T}$ and $\mathbf{g}=\left(s^{2}, t, t^{2}+s\right)^{T}$, respectively. First we reparameterize both surfaces $F$ and $G$ such that equal parameters correspond to parallel normals. With the normals $\mathbf{n}_{F}=(-8 v,-8 u, 16 u v)^{T}$ and $\mathbf{n}_{G}=(-1,-4 s t, 2 s)^{T}$ of $F$ and $G$ we find the affine versions of the Cremona transformations in the $[u, v]$-plane and in the $[s, t]$-plane, respectively:

$$
\varphi_{F}^{-1}: u=-\frac{1}{2 y}, \quad v=-\frac{1}{2 x} \quad \varphi_{G}^{-1}: s=-\frac{y}{2}, \quad t=-\frac{1}{2 x} .
$$

Inserting the latter equations into the parameterizations of $F$ and $G$ we obtain

$$
\mathbf{f}(x, y)=\frac{1}{2 x^{2} y^{2}}\left(y^{2}, x^{2},-2 x y(x+y)\right)^{T}, \quad \mathbf{g}(x, y)=\frac{1}{4 x^{2}}\left(1,-2 x y, x\left(x y^{2}-2\right)\right)^{T} .
$$



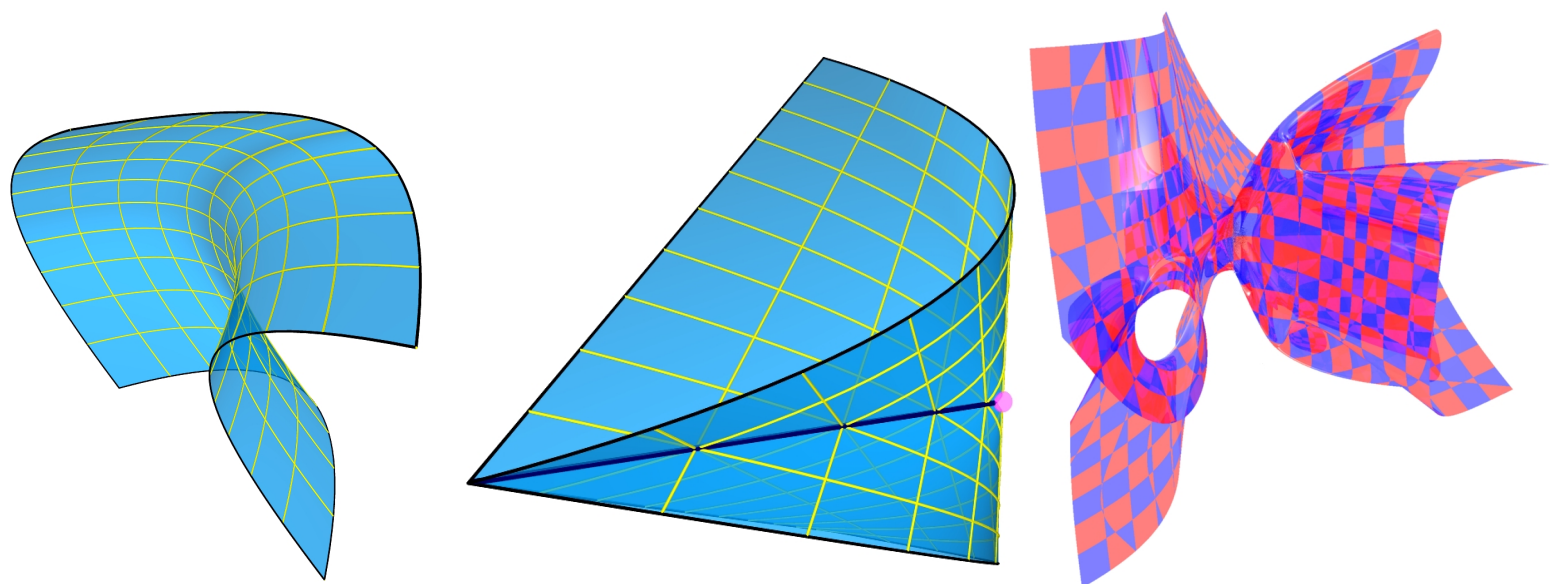

Figure 7: Convolution surface of two Steiner surfaces: $F$ (left), $G$ (middle), $C=F \star G$ (right).

A parameterization $\mathbf{c}$ of the convolution surface $C$ is thus computed as the sum of $\mathbf{f}$ and $\mathbf{g}$ as

$$
\mathbf{c}(x, y)=\frac{1}{4 x^{2} y^{2}}\left(3 y^{2}, 2 x^{2}\left(1-y^{3}\right), x y\left(x y^{3}-4 x-6 y\right)\right)^{T} .
$$

Fig. 8 shows the surfaces $F$ and $G$ as well as their convolution surface $C$.
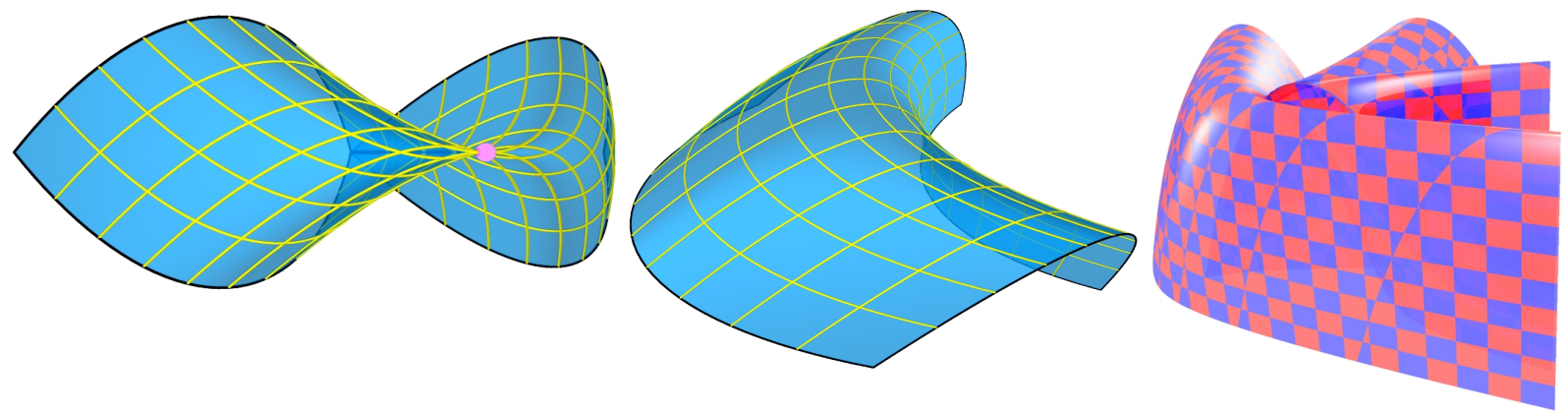

Figure 8: Convolution surface of two Steiner surfaces: $F$ (left), $G$ (middle), $C=F \star G$ (right).

\section{Conclusion}

We have given several geometric arguments which prove the LN-property of quadratic triangular Bézier surfaces. Planar Cremona transformations are used in order to straighten the parameter lines (conics) in the parameter plane. We have given several examples including convolution surfaces to illustrate the method. 
The LN-property in general can be characterized by the fact that the dual surface $S$ is a graph of a rational function which is equivalent to the fact that the plane at infinity is an $n-1$-fold tangent plane of surfaces $S$ of class $n$.

\section{Acknowledgments}

This work has been funded in parts by the Austrian Science Fund FWF within the research network S92.

\section{References}

[1] Albrecht, G., 2002. The Veronese surface revisited, J. Geom. 73, 22-38.

[2] Apery, F., 1987. Models of the Real Projective Plane: Computer Graphics of Steiner and Boy Surfaces. Vieweg, Braunschweig.

[3] Bloomenthal, J. and Shoemake, K., 1991. Convolution Surfaces, Computer Graphics, Vol. 25, No. 4, 251-256.

[4] Coffmann, A., Schwartz, A.J., Stanton, C, 1995. The algebra and geometry of Steiner and other quadratically parameterizable surfaces, Comp. Aided Geom. Design 13 (1996), $257-286$.

[5] Degen, W.L.F., 1994. The Types of Triangular Bézier Surfaces, in: IMA Conference on the Mathematics of Surfaces, 153-170.

[6] Farin, G., Hoschek, J., and Kim, M.-S., 2002. Handbook of Computer Aided Geometric Design, Elsevier.

[7] Fladt, K., 1933. Die Umkehrungen der ebenen quadratischen Cremona Transformationen, J. reine u. angew. Math. 170, 64-68.

[8] Hoschek, J., Lasser, D., 1993. Fundamentals of Computer Aided Geometric Design. A. K. Peters, Wellesley, MA 1993.

[9] Jüttler, B., 1998. Triangular Bézier surface patches with a linear normal vector field, in: The Mathematics of Surfaces VIII, Information Geometers, 431-446.

[10] Jüttler, B. and Sampoli, M.L., 2000. Hermite interpolation by piecewise polynomial surfaces with rational offsets, Comp. Aided Geom. Design 17, 361-385.

[11] Kummer, M., 1865. Über die Flächen vierten Grades, auf welchen Scharen von Kegelschnitten liegen, J. reine u. angew. Math. 64, 66-96. 
[12] Lávička, M., Bastl B. 2006. Rational parameterized curves and surfaces with rational convolutions. Algebraic Geometry and Geometric Modeling, Proc. of the conf., Barcelona, 4.-7. Sept. 2006, 74-79.

[13] Lee, I.-K., Kim, M.-S. and Elber, G., 1998. Polynomial/Rational Approximation of Minkowski Sum Boundary Curves, Graphical Models 60, No.2, 136-165.

[14] Lee, I.K., Kim, M.S. and Elber, G., 1998. The Minkowski Sum of 2D Curved Objects, Proceedings of Israel-Korea Bi-National Conference on New Themes in Computerized Geometrical Modeling, Feb.1998, Tel-Aviv University, 155-164.

[15] Meyer, W.F., 1903-1915. Spezielle algebraische Flächen. In: Enzyklopädie der Mathematischen Wissenschaften, Teubner, Leipzig, III C 10, 1483-1490, 1647-1660.

[16] Mühlthaler, H. and Pottmann, H., 2003. Computing the Minkowski sum of ruled surfaces, Graphical Models, 65, 369-384.

[17] Oeltze, S. and Preim, B., 2005, Visualization of Vasculature With Convolution Surfaces: Method, Validation and Evaluation, IEEE Transactions on Medical Imaging, Vol. 24, No.4, 540-548.

[18] Peternell, M. and Manhart, F., 2003. The convolution of a paraboloid and a parametrized surface, Journal for Geometry and Graphics 7, 157-171.

[19] Peternell, M. and Pottmann, H., 1998. A Laguerre geometric approach to rational offsets, Computer Aided Geometric Design 15, 223-249.

[20] Pottmann, H., 1995. Rational curves and surfaces with rational offsets, Computer Aided Geometric Design 12, 175-192.

[21] Pottmann, H., 1995. Studying NURBS curves and surfaces with classical geometry, in: Mathematical Methods for Curves and Surfaces, eds: M. Dæhlen and T. Lyche and L. L. Schumaker, Vanderbilt University Press, 413-438.

[22] Peters, J., Reif, U., 1998. The 42 equivalence classes of quadratic surfaces in affine $n$-space, Comp. Aided Geom. Design 15, 459-473.

[23] Sampoli, M.L., Peternell, M. and Jüttler, B., 2006. Exact parameterization of convolution surfaces and rational surfaces with linear normals, Computer Aided Geometric Design 23, 179-192.

[24] Schreier, O. and Sperner, E., 1961. Projective Geometry of $n$ Dimensions, Chelsea, NY.

[25] Sederberg, T.W., Anderson, D.C., 1985. Steiner surface patches, IEEE Comp. Graphics \& Applications 5, 23-36. 
[26] Sherstyuk, A., 1999. Convolution Surfaces in Computer Graphics. PhD thesis, Monash Univ., Australia.

[27] Steiner, J., 1882. Gesammelte Werke II, Berlin, 723-724, 741-742.

[28] Wunderlich, W., 1968. Durch Schiebung erzeugbare Römerflächen, Sitzungsberichte der Österreischen Akademie der Wissenschaften. 176, 473-497.

[29] Wunderlich, W., 1969. Kinematisch erzeugbare Römerflächen, J. reine u. angew. Math. 236, 67-78.

[30] Wunderlich, W., 1962. Römerflächen mit ebenen Fallinien, Ann. di Mat. pura ed appl. 57, 97-108. 